\title{
Crystal structure of 3-(2-chlorobenzoyl)-1,1-diphenylthiourea, $\left(\mathrm{C}_{6} \mathrm{H}_{5}\right)_{2} \mathrm{~N}(\mathrm{CS})(\mathrm{NH})(\mathrm{CO})\left(\mathrm{C}_{6} \mathrm{H}_{4} \mathrm{Cl}\right)$
}

\author{
G. Binzet ${ }^{1}$, U. Flörke ${ }^{I I}$, N. Külcü̈ ${ }^{I}$ and H. Arslan*,I \\ I Mersin University, Faculty of Arts and Sciences, Department of Chemistry, 33342 Mersin, Turkey \\ II Universität Paderbom, Fakultät für Naturwissenschaften, Department Chemie, Warburgerstr. 100, 33098 Paderborn, Germany
}

Received June 28, 2004, accepted and available on-line October 6, 2004; CCDC no. 1267/1359
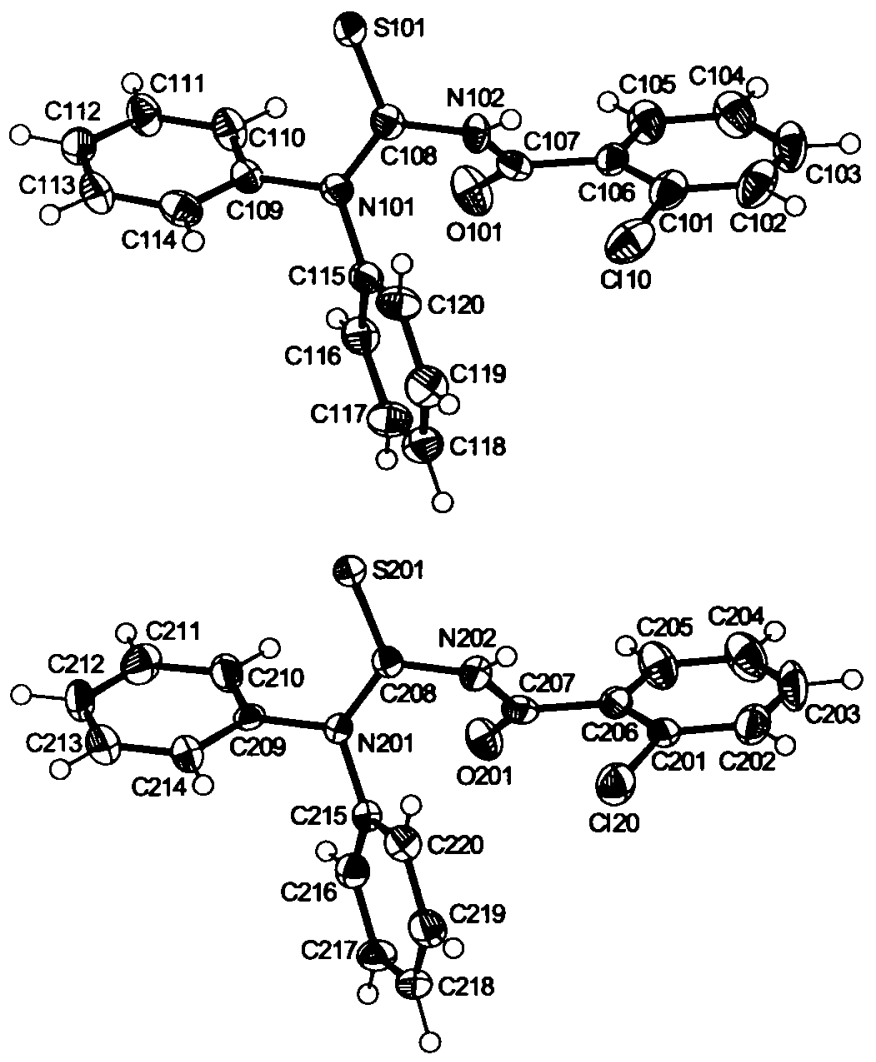

Discussion

The biological activities of thiourea derivatives have been successfully screened for various biological actions, and some derivatives have been used in commercial fungicides [1]. Also these derivatives have been found to be useful compounds for the enrichment and potential determination of transition metals from different matrices [3]. Recently, we discussed a novel series of thiourea derivatives and their metal complexes [4-7]. One of these new derivatives is also the title compound.

There are two independent, but identical molecules per asymmetric unit. The geometric parameters of the second molecule are very close to those of the first one, so the following discussion focuses only on the first molecule. The bond lengths of the carbonyl and thiocarbonyl groups have typical double-bond character $d(\mathrm{C} 107-0101)=1.214(4) \AA, d(\mathrm{C} 108-S 101) 1.657(4) \AA$. The bond lengths of all $\mathrm{C}-\mathrm{N}$ bonds $d(\mathrm{C} 115-\mathrm{N} 101)=1.445(4) \AA$, $d(\mathrm{C} 109-\mathrm{N} 101)=1.444(4) \AA, d(\mathrm{C} 108-\mathrm{N} 101)=1.347(4) \AA$, $d(\mathrm{C} 108-\mathrm{N} 102)=1.388(4) \AA, d(\mathrm{C} 107-\mathrm{N} 102)=1.387(4) \AA$ are shorter than the normal $\mathrm{C}-\mathrm{N}$ single-bond $(1.48 \AA)$ and longer than normal $\mathrm{C}=\mathrm{N}$ double bond $(1.25 \AA)$. These results are in agreement with expected delocalization in the molecule and confirmed by $\angle \mathrm{C} 107-\mathrm{N} 102-\mathrm{C} 108=123.9(3)^{\circ}, \angle \mathrm{C} 108-\mathrm{N} 101-$ $\mathrm{C} 109=120.4(3)^{\circ}$ showing an $s p^{2}$ hybridization on N101 and N102 atoms. The conformation of the molecule with respect to the thiocarbonyl and carbonyl moieties is twisted, as reflected by the torsion angles $\angle \mathrm{O} 101-\mathrm{C} 107-\mathrm{N} 102-\mathrm{C} 108$ and $\angle \mathrm{C} 107-$ $\mathrm{N} 102-\mathrm{C} 108-\mathrm{N} 101$ of $-4.2(6)^{\circ}$ and $48.8(5)^{\circ}$, respectively.

Abstract

$\mathrm{C}_{20} \mathrm{H}_{15} \mathrm{ClN}_{2} \mathrm{OS}$, triclinic, $P \overline{1}$ (no. 2), $a=9.9962(8) \AA$,

$b=12.911(1) \AA, c=14.670(1) \AA, \alpha=85.568(2)^{\circ}$,

$\beta=70.610(2)^{\circ}, \gamma=82.639(2)^{\circ}, V=1770.1 \AA^{3}, Z=4$, $R_{\mathrm{gt}}(F)=0.046, w R_{\mathrm{ref}}\left(F^{2}\right)=0.079, T=173 \mathrm{~K}$.

\section{Source of material}

All chemicals used for the preparation of the title compound were of reagent grade quality. The compound was prepared by a procedure similar to that reported in $[1,2]$. A solution of an appropriate 2-chlorobenzoyl chloride $(10 \mathrm{mmol})$ in acetone $(50 \mathrm{~mL})$ was added dropwise to a suspension of potassium thiocyanate $(10 \mathrm{mmol})$ in acetone $(30 \mathrm{~mL})$. The reaction mixture was heated under reflux for $30 \mathrm{~min}$, and then cooled to room temperature. A solution of diphenylamine $(10 \mathrm{mmol})$ in acetone $(10 \mathrm{~mL})$ was added and the resulting mixture was stirred for $2 \mathrm{~h} .0 .1 \mathrm{~N}$ Hydrochloric acid $(300 \mathrm{~mL})$ was added and the solution filtered. The solid product was washed with water and purified by recrystallization from ethanol.

\footnotetext{
* Correspondence author (e-mail: arslanh@mersin.edu.tr)
}

Table 1. Data collection and handling.

$\begin{array}{ll}\text { Crystal: } & \text { yellow prism, size } 0.12 \times 0.15 \times 0.20 \mathrm{~mm} \\ \text { Wavelength: } & \text { Mo } K_{\alpha} \text { radiation }(0.71073 \AA) \\ \mu: & 3.44 \mathrm{~cm}^{-1} \\ \text { Diffractometer, scan mode: } & \text { Bruker SMART CCD, } \varphi / \omega \\ 2 \theta_{\text {max }}: & 51.36^{\circ} \\ N(h k l)_{\text {measured, }} N(h k l)_{\text {hnique: }} & 9926,6594 \\ \text { Criterion for } I_{\text {obs, }} N(h k l)_{\text {gt: }}: & I_{\text {obs }}>2 \sigma\left(I_{\text {obs }}\right), 2600 \\ N(\text { param })_{\text {refined: }} & 451 \\ \text { Program: } & \text { SHELXTL [8] }\end{array}$

Table 2. Atomic coordinates and displacement parameters (in $\AA^{2}$ ).

\begin{tabular}{llllll}
\hline Atom & Site & $x$ & $y$ & $z$ & $U_{\text {iso }}$ \\
\hline H(10A) & $2 i$ & 0.6035 & 0.1013 & 0.4613 & 0.031 \\
H(10B) & $2 i$ & 1.0248 & 0.0911 & 0.5246 & 0.062 \\
H(10E) & $2 i$ & 0.9719 & 0.1081 & 0.6897 & 0.062 \\
H(10D) & $2 i$ & 0.7423 & 0.1710 & 0.7832 & 0.058 \\
H(10C) & $2 i$ & 0.5715 & 0.2272 & 0.7104 & 0.049 \\
H(11D) & $2 i$ & 0.1780 & 0.3186 & 0.5523 & 0.042
\end{tabular}


Table 2. Continued.

\begin{tabular}{llrlll}
\hline Atom & Site & \multicolumn{1}{l}{$x$} & $y$ & $z$ & $U_{\text {iso }}$ \\
\hline H(11E) & $2 i$ & -0.0530 & 0.3810 & 0.5542 & 0.045 \\
H(11I) & $2 i$ & -0.1050 & 0.4052 & 0.4110 & 0.042 \\
H(11B) & $2 i$ & 0.0727 & 0.3731 & 0.2650 & 0.044 \\
H(11A) & $2 i$ & 0.3055 & 0.3113 & 0.2609 & 0.042 \\
H(11F) & $2 i$ & 0.4431 & 0.4649 & 0.4198 & 0.040 \\
H(11G) & $2 i$ & 0.6345 & 0.5560 & 0.3301 & 0.047 \\
H(11H) & $2 i$ & 0.8222 & 0.4733 & 0.2062 & 0.045 \\
H(11C) & $2 i$ & 0.8163 & 0.3014 & 0.1746 & 0.048 \\
H(12A) & $2 i$ & 0.6287 & 0.2092 & 0.2649 & 0.042 \\
H(20A) & $2 i$ & 0.0072 & 0.4021 & -0.0733 & 0.030 \\
H(20B) & $2 i$ & -0.2672 & 0.4553 & -0.2705 & 0.049 \\
H(20D) & $2 i$ & -0.5027 & 0.4613 & -0.1681 & 0.060
\end{tabular}

Table 2. Continued.

\begin{tabular}{|c|c|c|c|c|c|}
\hline Atom & Site & $x$ & $y$ & $z$ & $U_{\text {iso }}$ \\
\hline $\mathrm{H}(20 \mathrm{E})$ & $2 i$ & -0.5546 & 0.3864 & -0.0148 & 0.061 \\
\hline $\mathrm{H}(20 \mathrm{C})$ & $2 i$ & -0.3734 & 0.3052 & 0.0412 & 0.051 \\
\hline $\mathrm{H}(21 \mathrm{D})$ & $2 i$ & 0.0833 & 0.1669 & 0.1735 & 0.041 \\
\hline $\mathbf{H}(21 \mathrm{H})$ & $2 i$ & 0.2093 & 0.0898 & 0.2746 & 0.052 \\
\hline$H(21 I)$ & $2 i$ & 0.4563 & 0.0497 & 0.2123 & 0.054 \\
\hline $\mathrm{H}(21 \mathrm{G})$ & $2 i$ & 0.5752 & 0.0882 & 0.0519 & 0.051 \\
\hline $\mathbf{H}(21 \mathrm{~F})$ & $2 i$ & 0.4507 & 0.1653 & -0.0494 & 0.041 \\
\hline$H(21 B)$ & $2 i$ & 0.2654 & 0.2752 & -0.1912 & 0.037 \\
\hline$H(21 E)$ & $2 i$ & 0.3016 & 0.1769 & -0.3274 & 0.044 \\
\hline $\mathbf{H}(21 \mathrm{C})$ & $2 i$ & 0.2582 & 0.0020 & -0.3074 & 0.042 \\
\hline$H(2 \mid A)$ & $2 i$ & 0.1748 & -0.0740 & -0.1532 & 0.041 \\
\hline$H(22 A)$ & $2 i$ & 0.1365 & 0.0235 & -0.0168 & 0.034 \\
\hline
\end{tabular}

Table 3. Atomic coordinates and displacement parameters (in $\AA^{2}$ ).

\begin{tabular}{|c|c|c|c|c|c|c|c|c|c|c|}
\hline Atom & Site & $x$ & $y$ & $z$ & $U_{11}$ & $U_{22}$ & $U_{33}$ & $U_{12}$ & $U_{13}$ & $U_{23}$ \\
\hline $\mathrm{Cl}(10)$ & $2 i$ & $0.8766(1)$ & $0.13886(9)$ & $0.39492(9)$ & $0.0336(7)$ & $0.0553(8)$ & $0.0573(9)$ & $-0.0087(6)$ & $-0.0016(6)$ & $-0.0109(7)$ \\
\hline$S(101)$ & $2 i$ & $0.3242(1)$ & $0.07885(7)$ & $0.46145(8)$ & $0.0301(6)$ & $0.0271(6)$ & $0.0453(8)$ & $-0.0041(5)$ & $-0.0179(6)$ & $0.0054(6)$ \\
\hline$O(101)$ & $2 i$ & $0.5051(3)$ & $0.3113(2)$ & $0.5564(2)$ & $0.060(2)$ & $0.031(2)$ & $0.043(2)$ & $0.013(2)$ & $-0.025(2)$ & $-0.012(2)$ \\
\hline$N(101)$ & $2 i$ & $0.4028(3)$ & $0.2690(2)$ & $0.4067(2)$ & $0.024(2)$ & $0.023(2)$ & $0.033(2)$ & $-0.001(2)$ & $-0.008(2)$ & $0.004(2)$ \\
\hline$N(102)$ & $2 i$ & $0.5447(3)$ & $0.1586(2)$ & $0.4784(2)$ & $0.021(2)$ & $0.022(2)$ & $0.036(2)$ & $0.009(2)$ & $-0.013(2)$ & $-0.008(2)$ \\
\hline$C(101)$ & $2 i$ & $0.8288(4)$ & $0.1487(3)$ & $0.5200(3)$ & $0.032(3)$ & $0.029(3)$ & $0.051(3)$ & $-0.004(2)$ & $-0.015(3)$ & $-0.008(2)$ \\
\hline$C(102)$ & $2 i$ & $0.9319(4)$ & $0.1185(3)$ & $0.5627(4)$ & $0.037(3)$ & $0.035(3)$ & $0.090(5)$ & $-0.004(2)$ & $-0.029(3)$ & $-0.015(3)$ \\
\hline$C(103)$ & $2 i$ & $0.9005(5)$ & $0.1280(3)$ & $0.6602(4)$ & $0.056(3)$ & $0.033(3)$ & $0.089(5)$ & $-0.004(3)$ & $-0.053(3)$ & $-0.006(3)$ \\
\hline$C(104)$ & $2 i$ & $0.7653(5)$ & $0.1665(3)$ & $0.7152(3)$ & $0.066(3)$ & $0.039(3)$ & $0.054(4)$ & $-0.007(3)$ & $-0.038(3)$ & $-0.002(3)$ \\
\hline$C(105)$ & $2 i$ & $0.6637(4)$ & $0.1983(3)$ & $0.6724(3)$ & $0.050(3)$ & $0.036(3)$ & $0.047(3)$ & $-0.006(2)$ & $-0.030(3)$ & $-0.005(3)$ \\
\hline$C(106)$ & $2 i$ & $0.6942(4)$ & $0.1886(3)$ & $0.5737(3)$ & $0.026(2)$ & $0.021(2)$ & $0.040(3)$ & $-0.004(2)$ & $-0.014(2)$ & $0.000(2)$ \\
\hline$C(107)$ & $2 i$ & $0.5760(4)$ & $0.2278(3)$ & $0.5343(3)$ & $0.033(3)$ & $0.022(2)$ & $0.030(3)$ & $-0.004(2)$ & $-0.011(2)$ & $0.005(2)$ \\
\hline$C(108)$ & $2 i$ & $0.4262(4)$ & $0.1743(3)$ & $0.4476(3)$ & $0.025(2)$ & $0.025(2)$ & $0.029(3)$ & $0.004(2)$ & $-0.006(2)$ & $-0.003(2)$ \\
\hline$C(109)$ & $2 i$ & $0.2616(4)$ & $0.3086(3)$ & $0.4055(3)$ & $0.029(2)$ & $0.019(2)$ & $0.025(3)$ & $0.002(2)$ & $-0.010(2)$ & $-0.004(2)$ \\
\hline$C(110)$ & $2 i$ & $0.1565(4)$ & $0.3294(3)$ & $0.4936(3)$ & $0.038(3)$ & $0.040(3)$ & $0.029(3)$ & $0.006(2)$ & $-0.015(2)$ & $-0.002(2)$ \\
\hline$C(111)$ & $2 i$ & $0.0200(4)$ & $0.3661(3)$ & $0.4945(3)$ & $0.035(3)$ & $0.038(3)$ & $0.036(3)$ & $0.009(2)$ & $-0.010(2)$ & $-0.001(2)$ \\
\hline$C(112)$ & $2 i$ & $-0.0105(4)$ & $0.3811(3)$ & $0.4098(3)$ & $0.033(3)$ & $0.032(3)$ & $0.044(3)$ & $-0.004(2)$ & $-0.018(2)$ & $0.002(2)$ \\
\hline$C(113)$ & $2 i$ & $0.0943(4)$ & $0.3616(3)$ & $0.3236(3)$ & $0.043(3)$ & $0.041(3)$ & $0.031(3)$ & $0.002(2)$ & $-0.020(2)$ & $0.003(2)$ \\
\hline$C(114)$ & $2 i$ & $0.2324(4)$ & $0.3249(3)$ & $0.3209(3)$ & $0.047(3)$ & $0.032(3)$ & $0.026(3)$ & $-0.005(2)$ & $-0.012(2)$ & $0.000(2)$ \\
\hline$C(115)$ & $2 i$ & $0.5187(4)$ & $0.3283(3)$ & $0.3521(3)$ & $0.027(2)$ & $0.027(2)$ & $0.026(3)$ & $-0.002(2)$ & $-0.007(2)$ & $0.003(2)$ \\
\hline$C(116)$ & $2 i$ & $0.5201(4)$ & $0.4311(3)$ & $0.3703(3)$ & $0.031(2)$ & $0.024(2)$ & $0.040(3)$ & $0.005(2)$ & $-0.008(2)$ & $-0.003(2)$ \\
\hline$C(117)$ & $2 i$ & $0.6331(4)$ & $0.4854(3)$ & $0.3167(3)$ & $0.047(3)$ & $0.024(2)$ & $0.047(3)$ & $-0.010(2)$ & $-0.015(3)$ & $-0.001(2)$ \\
\hline$C(118)$ & $2 i$ & $0.7442(4)$ & $0.4366(3)$ & $0.2434(3)$ & $0.034(3)$ & $0.038(3)$ & $0.038(3)$ & $-0.007(2)$ & $-0.008(2)$ & $0.002(2)$ \\
\hline$C(119)$ & $2 i$ & $0.7405(4)$ & $0.3348(3)$ & $0.2252(3)$ & $0.035(3)$ & $0.041(3)$ & $0.034(3)$ & $0.004(2)$ & $-0.002(2)$ & $-0.005(2)$ \\
\hline$C(120)$ & $2 i$ & $0.6295(4)$ & $0.2798(3)$ & $0.2784(3)$ & $0.042(3)$ & $0.023(2)$ & $0.033(3)$ & $-0.004(2)$ & $-0.005(2)$ & $-0.003(2)$ \\
\hline $\mathrm{Cl}(20)$ & $2 i$ & $-0.0061(1)$ & $0.37259(8)$ & $-0.25259(8)$ & $0.0429(7)$ & $0.0558(8)$ & $0.0304(7)$ & $-0.0002(6)$ & $-0.0124(6)$ & $0.0004(6)$ \\
\hline$S(201)$ & $2 i$ & $0.1433(1)$ & $0.40552(7)$ & $0.06473(8)$ & $0.0300(6)$ & $0.0259(6)$ & $0.0336(7)$ & $0.0001(5)$ & $-0.0150(5)$ & $-0.0065(5)$ \\
\hline$O(201)$ & $2 i$ & $-0.1080(2)$ & $0.1935(2)$ & $-0.0015(2)$ & $0.032(2)$ & $0.027(2)$ & $0.045(2)$ & $-0.004(1)$ & $-0.012(2)$ & $0.008(2)$ \\
\hline$N(201)$ & $2 i$ & $0.1771(3)$ & $0.2181(2)$ & $-0.0090(2)$ & $0.028(2)$ & $0.025(2)$ & $0.025(2)$ & $0.001(2)$ & $-0.014(2)$ & $-0.003(2)$ \\
\hline$N(202)$ & $2 i$ & $0.0058(3)$ & $0.3415(2)$ & $-0.0412(2)$ & $0.030(2)$ & $0.020(2)$ & $0.029(2)$ & $-0.008(2)$ & $-0.014(2)$ & $0.005(2)$ \\
\hline$C(201)$ & $2 i$ & $-0.1798(4)$ & $0.3780(3)$ & $-0.1733(3)$ & $0.028(2)$ & $0.026(2)$ & $0.038(3)$ & $0.002(2)$ & $-0.016(2)$ & $-0.011(2)$ \\
\hline$C(202)$ & $2 i$ & $-0.2887(4)$ & $0.4254(3)$ & $-0.2068(3)$ & $0.054(3)$ & $0.031(3)$ & $0.050(3)$ & $-0.003(2)$ & $-0.033(3)$ & $0.000(2)$ \\
\hline$C(203)$ & $2 i$ & $-0.4277(4)$ & $0.4283(3)$ & $-0.1463(4)$ & $0.036(3)$ & $0.046(3)$ & $0.080(4)$ & $0.000(2)$ & $-0.039(3)$ & $0.001(3)$ \\
\hline$C(204)$ & $2 i$ & $-0.4581(4)$ & $0.3844(3)$ & $-0.0557(4)$ & $0.024(3)$ & $0.051(3)$ & $0.075(4)$ & $-0.002(2)$ & $-0.013(3)$ & $-0.002(3)$ \\
\hline$C(205)$ & $2 i$ & $-0.3506(4)$ & $0.3365(3)$ & $-0.0221(3)$ & $0.029(3)$ & $0.040(3)$ & $0.056(3)$ & $-0.004(2)$ & $-0.011(3)$ & $0.008(3)$ \\
\hline$C(206)$ & $2 i$ & $-0.2096(4)$ & $0.3344(3)$ & $-0.0810(3)$ & $0.023(2)$ & $0.025(2)$ & $0.034(3)$ & $-0.005(2)$ & $-0.012(2)$ & $-0.005(2)$ \\
\hline$C(207)$ & $2 i$ & $-0.1006(4)$ & $0.2801(3)$ & $-0.0394(3)$ & $0.028(2)$ & $0.028(2)$ & $0.024(3)$ & $0.001(2)$ & $-0.004(2)$ & $-0.007(2)$ \\
\hline$C(208)$ & $2 i$ & $0.1096(3)$ & $0.3159(3)$ & $0.0030(3)$ & $0.019(2)$ & $0.025(2)$ & $0.021(2)$ & $-0.002(2)$ & $-0.003(2)$ & $0.000(2)$ \\
\hline$C(209)$ & $2 i$ & $0.2558(4)$ & $0.1739(3)$ & $0.0532(3)$ & $0.033(2)$ & $0.017(2)$ & $0.023(3)$ & $-0.002(2)$ & $-0.013(2)$ & $-0.003(2)$ \\
\hline$C(210)$ & $2 i$ & $0.1840(4)$ & $0.1511(3)$ & $0.1486(3)$ & $0.032(2)$ & $0.036(3)$ & $0.034(3)$ & $0.004(2)$ & $-0.014(2)$ & $0.001(2)$ \\
\hline$C(211)$ & $2 i$ & $0.2586(4)$ & $0.1051(3)$ & $0.2085(3)$ & $0.065(3)$ & $0.035(3)$ & $0.033(3)$ & $-0.007(2)$ & $-0.023(3)$ & $0.007(2)$ \\
\hline$C(212)$ & $2 i$ & $0.4047(5)$ & $0.0816(3)$ & $0.1717(4)$ & $0.051(3)$ & $0.035(3)$ & $0.061(4)$ & $0.010(2)$ & $-0.040(3)$ & $-0.004(3)$ \\
\hline$C(213)$ & $2 i$ & $0.4746(4)$ & $0.1042(3)$ & $0.0770(3)$ & $0.035(3)$ & $0.043(3)$ & $0.052(3)$ & $0.006(2)$ & $-0.020(3)$ & $-0.008(3)$ \\
\hline$C(214)$ & $2 i$ & $0.4010(4)$ & $0.1502(3)$ & $0.0168(3)$ & $0.028(2)$ & $0.038(3)$ & $0.039(3)$ & $0.005(2)$ & $-0.015(2)$ & $-0.005(2)$ \\
\hline$C(215)$ & $2 i$ & $0.1956(4)$ & $0.1588(3)$ & $-0.0931(3)$ & $0.023(2)$ & $0.026(2)$ & $0.026(3)$ & $-0.000(2)$ & $-0.010(2)$ & $-0.002(2)$ \\
\hline$C(216)$ & $2 i$ & $0.2459(4)$ & $0.2043(3)$ & $-0.1835(3)$ & $0.029(2)$ & $0.032(2)$ & $0.029(3)$ & $-0.004(2)$ & $-0.006(2)$ & $0.001(2)$ \\
\hline$C(217)$ & $2 i$ & $0.2683(4)$ & $0.1457(3)$ & $-0.2643(3)$ & $0.034(3)$ & $0.045(3)$ & $0.025(3)$ & $0.005(2)$ & $-0.004(2)$ & $-0.001(2)$ \\
\hline$C(218)$ & $2 i$ & $0.2422(4)$ & $0.0422(3)$ & $-0.2524(3)$ & $0.035(3)$ & $0.041(3)$ & $0.029(3)$ & $0.007(2)$ & $-0.011(2)$ & $-0.014(2)$ \\
\hline$C(219)$ & $2 i$ & $0.1930(4)$ & $-0.0028(3)$ & $-0.1609(3)$ & $0.038(3)$ & $0.028(2)$ & $0.040(3)$ & $-0.004(2)$ & $-0.014(2)$ & $-0.010(2)$ \\
\hline$C(220)$ & $2 i$ & $0.1696(3)$ & $0.0547(3)$ & $-0.0800(3)$ & $0.026(2)$ & $0.031(2)$ & $0.028(3)$ & $-0.003(2)$ & $-0.008(2)$ & $0.000(2)$ \\
\hline
\end{tabular}


Acknowledgment. This work was supported by the Mersin University Research Fund (project nos.: FEF.K.HA.99.1, ECZ.F.TB.HA.2003.1 and FBE.KB.GP.01).

\section{References}

1. Polat, G.: Synthesis and characterization of novel thiourea ligands and their $\mathrm{Ni}(\mathrm{II}) . \mathrm{Cu}(\mathrm{II})$ and $\mathrm{Co}$ (II) complexes. MSc Thesis. Mersin University, Mersin. Turkey 2003.

2. Binzet. G.; Arslan, H.; Flörke, U.; Külcü, N.: Synthesis, characterization and crystal structures of some transition metal complexes with $N, N$ dialkyl- $N^{\prime}$-(2-chloro-benzoyl)thiourea derivatives. Transition Met. Chem., submitted 2004.

3. Irving, A.; Koch, K. R.; Matoetoe, M.: Deceptively simple Pt complexes of $N, N$-dialkyl- $N$ "-benzoylthiourea: a ${ }^{1} \mathrm{H},{ }^{15} \mathrm{C}$ and ${ }^{195} \mathrm{Pt}$ NMR study of their acid-base chemistry in solution and the molecular structure of cisbis( $N ; N$-di( $n$-butyl)- $N^{\prime}$-benzoylthioureato)platinum(II). Inorg. Chim. Acta. 206 (1993) 193-199.
4. Arslan, H.; Flörke, U.; Külcü, N.: Synthesis and characterization of copper(II), nickel(II) and cobalt(II) complexes with some novel thiourea derivatives. Transition Met. Chem. 28 (2003) 816-819.

5. Avsar, G.; Arslan, H.; Haupt, H.-J.; Külcü, N.: Crystal structure of cisbis $\left(N, N\right.$-dimethyl- $N^{\prime}$-benzoylthioureato)palladium(II). Turk. J. Chem. 27 (2003) 281-285

6. Arslan, H.; Flörke, U.; Külcü, N.: Synthesis, characterization and crystal structure of 1-(4-Chloro-benzoyl)-3-naphthalen-1-yl-thiourea. J. Chem. Crystallogr. 33 (2003) 919-924

7. Emen, F. M.: Flörke, U.; Külcü, N.; Arslan, H.: $c i s-B i s\left(N, N\right.$-dimethyl- $N^{\prime}-$ 2-chlorobenzoylthioureato)nickel(II). Acta Crystallogr. E59 (2003) m582-m583.

8. Sheldrick, G. M.: SHELXTL v. 5.1. Structure determination software suite, Bruker AXS, Madison, Wisconsin, USA 1998. 